\title{
Effect of lamination parameters on deformation energy of LTCC substrate based on Finite element analysis
}

\author{
Liyu $\mathrm{Li}^{\mathrm{a}}$, Zhaohua Wu ${ }^{\mathrm{b}, *}$ \\ Electromechanical Engineering College, Guilin University of Electronic Technology, Guilin, 541004, \\ China \\ agbfly2015@163.com, bemezdj@guet.edu.cn
}

Keywords: LTCC; Finite Element Analysis; Process Parameter; Deformation Energy.

\begin{abstract}
In this paper, the temperature, time and pressure of the three process parameters were selected as three control factors of the low temperature co fired ceramic (LTCC) substrate laminating process. By using an L9 (33) orthogonal array which have 9 different process parameters' levels combinations were designed. The finite element analysis model of the LTCC substrate were established by using the finite element software ANSYS. The LTCC substrate was analyses by the method that thermal structural coupled finite element analysis under the different process parameters. The substrate deformation energy was calculated. Based on the deformation energy of different process parameters combination, the range analysis was performed. The results show that temperature is the dominant factor in effecting on deformation energy, holding time secondly and pressure at last; temperature $70{ }^{\circ} \mathrm{C}$, time $10 \mathrm{~min}$ and pressure $25 \mathrm{Mpa}$ can reduce the substrate deformation and obtain excellent interlayer bonding effect. Combined with the references experimental results, the authors validate the conclusions are correct.
\end{abstract}

\section{Introduction}

Low temperature co-firing ceramic (LTCC) technology is an advanced passive integration and hybrid circuit packaging technology, it cannot only Package the resistor, capacitor and inductor, but also packaged by the IC integrated circuit module to be a complete circuit system. Because of the advantages of high frequency and high speed data communication, passive component and active component integration, high reliability, LTCC technology become the focus [1]. Low temperature co-fired ceramic (LTCC) substrate manufacturing process's lamination parameters includes temperature, pressure and time, however due to the combined effect of temperature, time and pressure, the laminated green tapes will produce deformation and delamination. The deformation and delamination will cause vertical interconnection dislocation and influence the electrical properties. Because of the uneven force and great material liquidity, the three-dimensional structures (such as surface channel, chamber, etc.) which embedded in multi-layer green tape often collapse [2].Analysis of lamination process characteristics, the green tape pressing quality is affected by temperature, pressure and time. To estimate the forming quality intuitively, this paper put forward with the deformation energy to estimate the index of forming quality. Then the lamination process was simulated based on orthogonal experiment. Finally, the authors analyzed and summed up the law of lamination process parameters effect on the green tapes so that achieve the purpose of guiding production.

\section{Warm Water Isotactic Pressing Technology Introduction}

This study research the lamination process which base on the warm water isostatic pressing technology. Warm water isostatic pressing technology mainly include stack, packaging, preheating, boosting, Holding pressure, decompression[3].Firstly, put the laminated green tapes into the vacuum bags. Then the packaged green ceramic tapes are vacuum. Finally, put the bag piece into the lamination cavity to laminate. The laminated green tapes structure is shown as Fig.1. Green tapes combined effects of temperature and pressure in water environment will soften and closely integrate 
other green tape. After holding pressure and pressure relief process, the green tapes is removed from the laminated cavity to complete the lamination process.

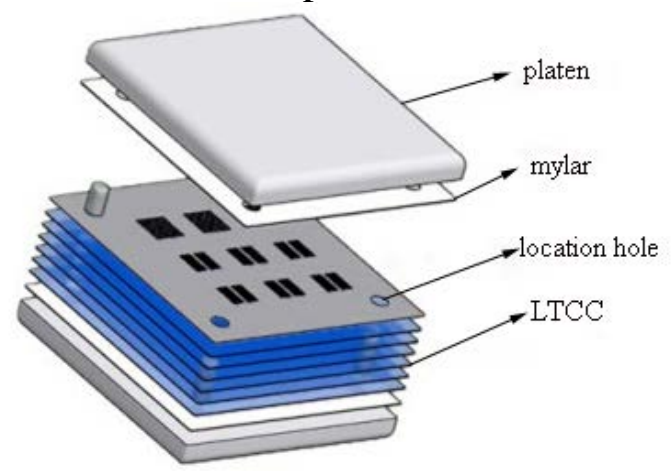

Fig.1 The laminated green tapes structure

\section{Finite Element Modeling}

In this paper, the substrate with 3D micro-channel structure embedded is composed of 26 layers of ferro-A6 green tape, and the dimensions of the green tapes is $40 \mathrm{~mm}$ by $50 \mathrm{~mm}$ by $0.127 \mathrm{~mm}$. The dimensions of the main runner is $36 \mathrm{~mm}$ by $2.2 \mathrm{~mm}$, and the dimensions of branch runner is $30.2 \mathrm{~mm}$ by $1 \mathrm{~mm}$. The sacrificial material is embedded into the micro-channel. The dimensions of sacrificial material is $30.1 \mathrm{~mm}$ by $0.9 \mathrm{~mm}$. Combined with requirements of RF function module dimensions and neglect of the effect of conductor on the deformation energy, corresponding three-dimensional geometric model is established. The overall geometry model as shown in Fig.2, the micro-channel sacrificial material model as shown in Fig.3, relevant dimensions are summarized in table 1.

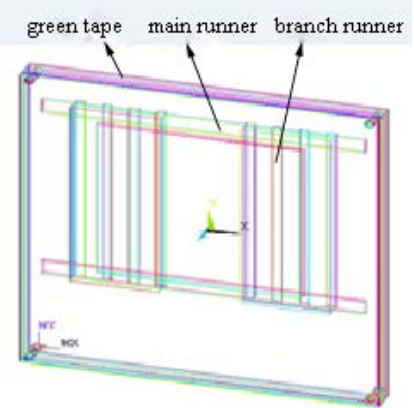

Fig.2. overall geometry model

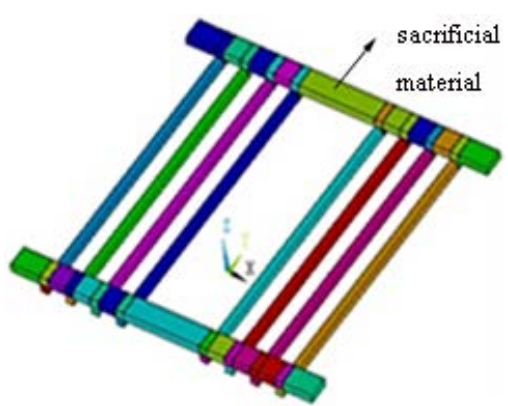

Fig.3. Micro-channel sacrificial material model Table 1.The geometric size of the model

\begin{tabular}{ccccc}
\hline dimension & green tape & main runner & branch runner & sacrificial material \\
\hline Length $(\mathrm{mm})$ & 50 & 36 & 30.2 & 30.1 \\
Wide $(\mathrm{mm})$ & 40 & 2.2 & 1 & 0.9 \\
Thickness $(\mathrm{mm})$ & 3.302 & 0.508 & 0.508 & 0.508 \\
\hline
\end{tabular}

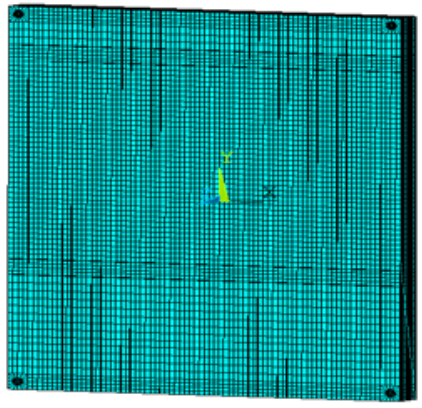

Fig.4 Finite element model of green tapes

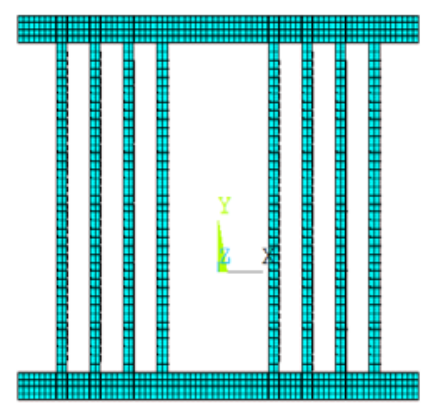

Fig.5 Finite element model of sacrificial material 
The simulation analysis use the finite element analysis software ANSYS, the 185 unit can be used to simulate the viscoelastic mechanical behavior. Carries on the grid division of the geometric model, finite element models of green tapes is shown in Fig.4, finite element models of sacrificial material is shown in fig.5.

The green tapes are made by ferro-A6.From the references and related mechanical experiment, using the Maxwell stress relaxation model can accurately describe the mechanical properties of this materials [4]. The stress function is $\sigma=\sigma_{0} e^{-t / 350}$, the curve is shown in Fig.6. The load curve of temperature and pressure is shown in Fig.7 [5].
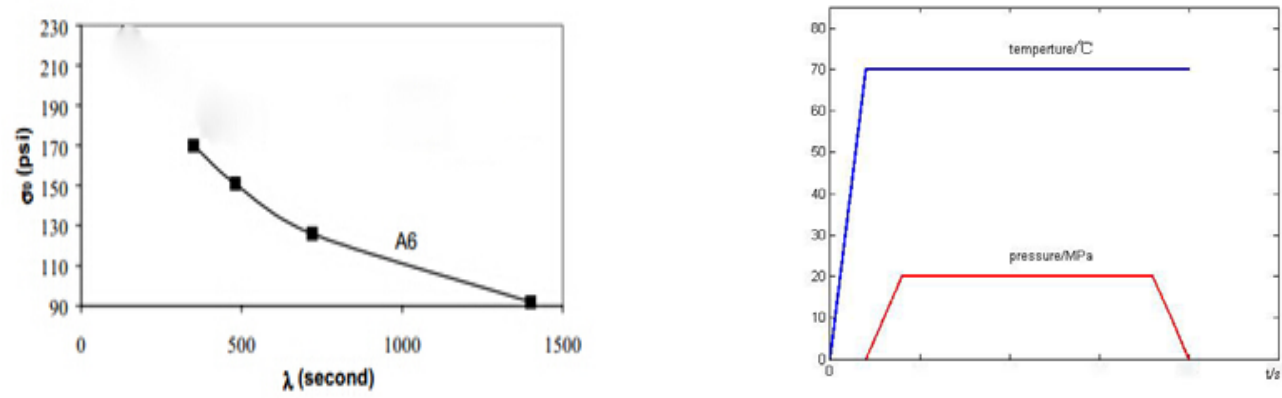

Fig.6 Ferro-A6 stree change with relaxation time Fig.7 Load curve of temperature and pressure

\section{Simulation Calculation and Results Discussion}

Using the indirect method of thermal structure coupled to simulate lamination process and obtain calculation results. Laminated integral deformation diagram is shown in Fig.8, the overall displacement contours is shown in Fig.9. Fig.8 shows that green tapes under each surface pressure lead to surrounding extrusion. The biggest displacement can be seen in the edge part, and the result present certain gradient from edge towards the center as shown in the contours of Fig.9. The green tapes was squeezed by the loads inevitably will expand along four sides [2], but the extent of deformation is small. Combined with experimental results of references 2, correct simulation can be seen from the deformation and displacement contours as shown in Fig.8 and Fig.9.Based on the orthogonal experiment, the lamination process is simulated and obtain the results. The deformation energy was calculated according to equation1, the calculated results are presented in table 2.
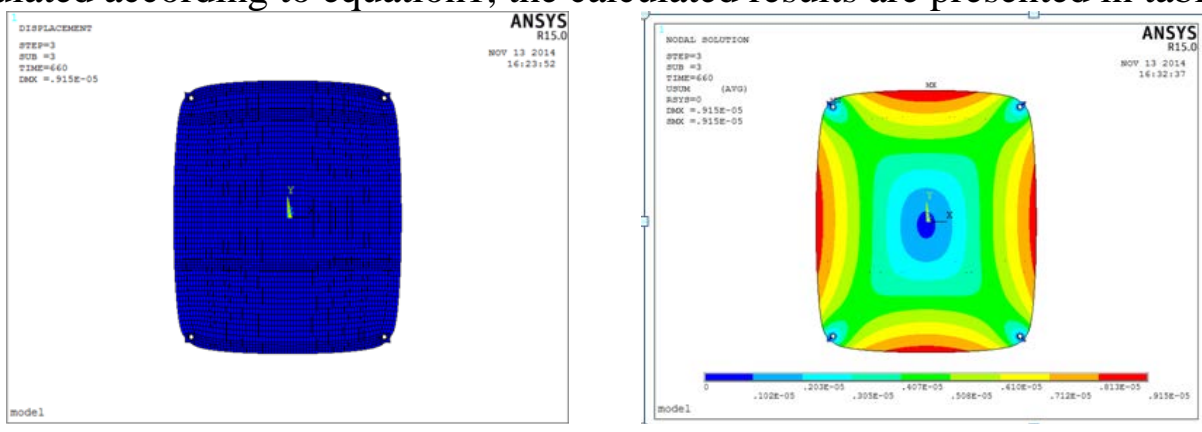

Fig.8 Laminated integral deformation diagram Fig.9 The overall displacement contours

$$
W=\sum_{k=1}^{n} \frac{1}{2}\left[\left(\sigma_{x} \varepsilon_{x}+\sigma_{y} \varepsilon_{y}+\sigma_{z} \varepsilon_{z}+\tau_{x y} \gamma_{x y}+\tau_{y} \gamma_{y z}++\tau_{z x} \gamma_{z x}\right)\right]_{k}
$$

In this equation, $\mathrm{k}$ is $\mathrm{k}$ element; $\mathrm{n}$ is the total number of element.

From the table.2, we can see that temperature is the dominant factor in effecting on deformation energy, holding time secondly and pressure at last. Pressure from 10Mpa to 30Mpa is relatively small effect on the deformation energy. Plastic deformation will happen so that the green tapes flows under the temperature and time. The green tapes want to buffer deformation to float all around. The higher pressure, the higher binding force between the green tapes [6], and Sintering shrinkage rate of the substrate decreased linearly decreased linearly [7]. In order to obtain a better combination between layers and avoid excessive pressure to damage the embedded structure, the authors recommend $25 \mathrm{Mpa}$ to be used in the process. 
Table. 2 The results of deformation energy

\begin{tabular}{ccccc}
\hline & $\mathrm{P}(\mathrm{Mpa})$ & $\mathrm{T}\left({ }^{\circ} \mathrm{C}\right)$ & $\mathrm{t}(\mathrm{min})$ & deformation energy $(\mathrm{J})$ \\
\hline 1 & 10 & 50 & 5 & 0.0174 \\
2 & 10 & 70 & 10 & 0.0287 \\
3 & 10 & 90 & 15 & 0.0436 \\
4 & 20 & 50 & 10 & 0.0146 \\
5 & 20 & 70 & 15 & 0.0264 \\
6 & 20 & 90 & 5 & 0.0565 \\
7 & 30 & 50 & 15 & 0.0135 \\
8 & 30 & 70 & 5 & 0.0342 \\
9 & 30 & 90 & 10 & 0.0475 \\
$\mathrm{~K}_{1 \mathrm{j}}$ & 0.0299 & 0.0152 & 0.0360 & \\
$\mathrm{~K}_{2 \mathrm{j}}$ & 0.0325 & 0.0297 & 0.0303 & \\
$\mathrm{~K}_{3 \mathrm{j}}$ & 0.0317 & 0.0492 & 0.0278 & \\
$\mathrm{R}$ & 0.0026 & 0.0340 & 0.0082 &
\end{tabular}

Note: $K_{\mathrm{ij}}$ is the sum of corresponding data in $\mathrm{i}$ level and $\mathrm{j}$ row; $\mathrm{R}$ is factor range.

\section{Conclusion}

This paper used finite element analysis to simulate LTCC lamination process. With that method, the authors obtained different deformation energy under different process parameters. In the light of process parameters and deformation energy analysis, the following conclusion are obtained.

(1) Pressure from 10Mpa to 30Mpa have litter effect on deformation. But in order to get excellent combination between green tapes, the authors suggest to use pressure $25 \mathrm{Mpa}$.

(2) In order to ensure a better adhesive effect between layers and avoid lashings of material liquidity, the authors suggest to use temperature $70^{\circ} \mathrm{C}$.

(3) Under the associated effects of temperature and time, the green tapes will flow around.

(4) temperature is the dominant factor in effecting on deformation energy, holding time secondly and pressure at last.

\section{Acknowledgement}

This work was supported by basic research projects “The Study of Multi-energy.

\section{References}

[1] Jianfen He. LTCC Substrate manufacture and control[J]. Electronics Process Technology, 2005 (02):75-81.

[2] Qing Liu, Jian Qin, xi Chen. Thermo-compression lamination of multilayer ceramic green tapes[J]. Bulletin of the Chinese ceramic society,2011,30(1):34-38.

[3] JiaoYan Jinzhong. Multilayer LTCC technology [M]. Beijing Science Publishing , 2010: 150.

[4] W.kinzy,Jones, Yangqingliu,BrooksLarsen,PengWang,andMarcZampino.Chemical,structural and mechanical properties of the LTCC tapes. The International Journal of Microcircuits and Electronic Packaging, Volume 23, Number 4, Fourth Quarter, 2000 (ISSN 1063-1674).

[5] Xiaoyao Li, Zhe Feng, Jianhong Zhao. The research of lamination process and the development of machine[J]. Equipment for Electronic Products Manufactturing,2012(10):24-26.

[6] Dong Zhou, Shaohong Wang,Zhaoxia Hou. Research progress in the lamination process of multilayered ceramic green tapes[J]. Ordnance Material science and Engineering,Vol.35.No4.

[7] Zhaowen Dong. Research of LTCC substrate manufacture process[J]. Electronic Components \&Materials,1998(05): 26-28+30. 